\title{
The Global Impact of Diabetes and Depression: A Brief Review
}

\author{
Sharon H Murff* \\ Associate Professor of Nursing, Grambling State University, USA
}

*Corresponding author: Sharon H Murff, Associate Professor of Nursing, Grambling

Received Date: October 02, 2019

State University, USA.

Published Date: October 14, 2019

\begin{abstract}
Diabetes is considered to be the seventh leading cause of death, globally. For several decades the worldwide incidence of diabetes has escalated. There are over 400 million people worldwide with a diagnosis of diabetes. Complications associated poor management of diabetes is also costly. In fact, the worldwide expense to care for people with diabetes has been estimated to be over eight billion U.S. dollars. People with diabetes often have comorbid conditions such as depression. In fact, it has been estimated that those with diabetes have a two times greater risk of being diagnosed with depression. Greater mortality is projected for people with a diagnosis of both diabetes and depression. In addition, there is an increase in the cost to care for those with comorbid conditions of diabetes and depression. Since diabetes and depression are costly conditions to manage, the design of innovative programs to prevent and effectively manage the conditions should be beneficial.
\end{abstract}

\section{Introduction}

Globally, the prevalence of diabetes has continued to increase over the past several decades. According to the World Health Organization [1], from 1980 to 2014 the number of people with diabetes has increased by over 300 million. In 1980 diabetes affected approximately 100 million people and in 2014 the number of those with diabetes was over 420 million (WHO, 2016). The mortality rate related to diabetes has also increased. Diabetes was the cause of death for over one million people worldwide and another two million deaths were related to hyperglycemia in 2012 (WHO, 2016). Globally, it has been estimated that diabetes is the seventh leading cause of death (WHO, 2016).

Diabetes is a metabolic disorder that involves pancreatic function. One of the chief hormones produced by the pancreas is insulin. One major pathological problem with diabetes is that insulin is not being produced in an adequate amount to handle the metabolic processes of the body. Another pathophysiological issue with diabetes is that the body is not effective in the utilization of the insulin that is being produced. If an adequate amount of insulin is not readily available for the body to use, glucose cannot enter the cell through the proper mechanism. As a result of pancreatic or insulin dysfunction, when glucose cannot enter the cell there is a subsequent increase in the amount of glucose in the bloodstream with possible life-threatening problems [2].
There are a number of complications that occur due to diabetes. Cardiovascular diseases such as myocardial infarction and stroke, kidney disease, visual disturbances that can lead blindness, nerve damage, and amputation of limbs are complications that can occur in persons with diabetes (Lewis et al., 2017). Diabetes, along with other comorbid conditions have been responsible for an increased mortality rate especially among those 70 years old or less. Also, mortality rates have increased at a more rapid pace in low to middle income countries compare to high income countries (WHO, 2016).

In addition to the increased risks from complications and death related to diabetes, there is an increase in the global burden of diabetes with respect to health expenditures. Included in the cost to care for people with diabetes are direct medical care costs, care for outpatient and inpatient services, and emergency department services. Also, people with diabetes incur expenses for supplies for self-monitoring devices, injections, and medications. The approximate worldwide expenditure to manage people with diabetes is over eight billion U.S. dollars (WHO, 2016). Depression is another disorder that has plagued countries. There are greater than 300 million people with depression worldwide and it affects people in all age groups. From a global perspective, depression has been identified as the principal cause of disability [3]. 
A connection between stress and chronic illnesses has been identified in the literature [4]. Likewise, Selye (1974) reported that there is a definite link between stress and disease. More specifically, it has been reported that there is an association between diabetes, depression, and the intensification of the risk for death [5].

People with diabetes have an almost two-fold risk of a diagnosis of depression compared to those without a diagnosis of diabetes (van Dooren et al., 2013). Among individuals with a diagnosis of diabetes and depression there is an increase in the use of primary care and emergency services. Also, people with the comorbid conditions of diabetes and depression have more prescription medicines than those without the comorbidities [6].

As previously indicated, the cost to care for people diabetes is high. The addition of a comorbid diagnosis of depression the cost to manage people with diabetes could be compounded. In other words, the cost to care for those with diabetes is already increased and coupled with the diagnosis of depression, more money is needed for proper care management. Continued research is needed to determine the reasons for the increased incidence of diabetes. Also, researchers should examine the reasons for the increased risk of a diagnosis of depression among those with diabetes. Therefore, more research is in order to identify astute strategies to effectively manage people with a diagnosis of depression and diabetes, for more judicious allocation of health care dollars.

\section{Acknowledgement}

None.

\section{Conflicts of Interest}

No conflicts of interest.

\section{References}

1. WHO (2016) Global report on diabetes.

2. Lewis SL, Bucher L, Heitkemper MM, Harding MM, Kwsong J et al. (2017) Medical-Surgical Nursing (10th ed.) St. Louis: Elsevier.

3. WHO (2018) Depression: Key facts.

4. Holmes T, Rahe R (1967) The social readjustment rating scale. Journal of Psychosomatic Research 11(2): 213-218.

5. Van Dooren FEP, Nefs G, Schram MT, Verhey FRJ, Denollet J (2013) Depression and risk of mortality in people with diabetes Mellitus: A systematic review and meta-analysis. PLoS One 8(3): 1-9 e57058.

6. Kalsekar ID, Madhavan SM, Amonkar MM, Scott V, Douglas SM et al. (2006) The effect of depression on utilization and costs in patients with type 2 diabetes. Managed Care Interface19(3): 39-46. 